\title{
VDIPEN, A Metalloproteinase-generated Neoepitope, Is Induced and Immunolocalized in Articular Cartilage during Inflammatory Arthritis
}

Irwin I. Singer, * Douglas W. Kawka, * Ellen K. Bayne," Susan A. Donatelli, „ Jeffrey R. Weidner, ${ }^{*}$ Hollis R. Williams, * Julia M. Ayala, * Richard A. Mumford, ${ }^{*}$ Michael W. Lark, ${ }^{*}$ Tibor T. Glant, ${ }^{\star}$ Gerald H. Nabozny, ${ }^{\mathbf{s}}$ and Chella S. David

"Division of Immunology and Inflammation, Merck Research Laboratories, Merck \& Co., Inc., Rahway, New Jersey 07065;

${ }^{\ddagger}$ Departments of Biochemistry and Orthopedic Surgery, Rush-Preysbyterian-St. Luke's Medical Center, Chicago, Illinois 60612; and ${ }^{8}$ Department of Immunology, Mayo Clinic, Rochester, Minnesota 55905

\section{Abstract}

The destruction of articular cartilage in immune inflammatory arthritic disease involves the proteolytic degradation of its extracellular matrix. The role of activated matrix metalloproteinases (MMPs) in the chondrodestructive process was studied by identifying a selective cleavage product of aggrecan in murine arthritis models initiated by immunization with either type II collagen or proteoglycan. We conducted semiquantitative immunocytochemical studies of VDIPEN $^{341}$ using a monospecific polyclonal antibody requiring the free $\mathrm{COOH}$ group of the $\mathrm{COOH}$-terminal Asn for epitope detection. This antibody recognizes the aggrecan G1 domain fragment generated by MMP [i.e., stromelysin (SLN) or gelatinase A] cleavage of aggrecan between Asn $^{341}$-Phe ${ }^{342}$ but does not recognize intact aggrecan. VDIPEN was undetectable in normal mouse cartilage but was observed in the articular cartilage (AC) of mice with collagen-induced arthritis $\mathbf{1 0} \mathbf{d}$ after immunization, without histological damage and clinical symptoms. This aggrecan neoepitope was colocalized with high levels of glycosaminoglycans (GAGs) in pericellular matrices of $\mathrm{AC}$ chondrocytes but was not seen at the articular surface at this early time. Digestion of normal (VDIPEN negative) mouse paw cryosections with SLN also produced heavy pericellular VDIPEN labeling. Computer-based image analysis showed that the amount of VDIPEN expression increased dramatically by 20 d (70\% of the SLN maximum) and was correlated with GAG depletion. Both infiltration of inflammatory cells into the synovial cavity and early $\mathrm{AC}$ erosion were also very prominent at this time. Analysis of adjacent sections showed that both induction of VDIPEN and GAG depletion were strikingly codistributed within sites of articular cartilage damage. Similar results occurred in proteoglycan-induced arthritis, a more progressive and chronic model of inflammatory arthritis. These studies demonstrate for the first time the MMP-dependent catabolism of aggrecan at sites of chondrodestruction during inflammatory arthritis. (J. Clin. Invest. 1995. 95:2178-2186.) Key words: arthritis • articular cartilage $\bullet$ aggrecan $\bullet$ neoepitope $\bullet$ immunostaining

Address correspondence to I. I. Singer, Ph.D., Merck Research Laboratories, Merck \& Co., Inc., P.O. Box 2000, Rahway, NJ 07065. Phone: 908-594-5574; FAX: 908-594-3111.

Received for publication 25 August 1994 and in revised form 12 December 1994.

The Journal of Clinical Investigation, Inc.

Volume 95, May 1995, 2178-2186

\section{Introduction}

RA is a chronic joint disease characterized by articular cartilage (AC) ${ }^{1}$ destruction and synovial membrane inflammation associated with loss of joint function. Cartilage erosion results from cleavage of its dominant extracellular matrix (ECM) components: large aggregating proteoglycan (aggrecan) and type II collagen (CII). Aggrecan is a high buoyant density proteoglycan (PG) consisting of a protein core $\left(M_{\mathrm{r}} 2.0-2.5 \times 10^{5}\right)$ to which chondroitin sulfate and keratan sulfate glycosaminoglycan (GAG) side chains and $\mathrm{N}$-linked and $\mathrm{O}$-linked oligosaccharides are attached $(1,2)$. The core protein contains three globular domains (G1, G2, and G3) and an interglobular domain (between $\mathrm{G} 1$ and G2). The G1 domain mediates the binding of aggrecan to hyaluronan and the formation of large aggregates that are stabilized by link protein. Several matrix metalloproteinases (MMPs) have been implicated in the catabolism of cartilage ECM: stromelysin (SLN; EC 3.4.24.17), collagenase (CLN; EC3.4.24.7), gelatinase A (GLN-A; EC3.4.24.24), and gelatinase B (GLN-B; EC3.4.24.35) (3-6). SLN appears to be important in both inflammatory and degenerative arthritides, because it can directly degrade aggrecan (7) and link protein (8), type II collagen telopeptide, and type IX collagen (9), is elevated in synovial fluids and cartilage of RA and osteoarthritic (OA) patients, and may be up-regulated in synoviocytes and chrondrocytes (10-14).

Experimental animal models of inflammatory arthritis have provided important insights for understanding human arthritis. Although the pathological mechanisms of type II collagen-induced (CIA) and PG-induced (PGIA) arthritis are different and not fully understood, both appear to be sustained by genetically controlled autoimmune responses to cartilage ECM components (15-21). These models share many similarities with human RA and ankylosing spondylitis, such as humoral and cellular immune responses to immunizing and self-antigens, mononuclear cell infiltration of the synovium, formation of an erosive pannus, and progressive loss of PG and destruction of articular cartilage $(18-20,22)$. The pathogenesis of CIA and PGIA appears to be dependent on binding of PG or CII antibodies to joint cartilage followed by C5a release (23-25), activation of autoreactive $\mathrm{T}$ cells to matrix components $(21,26-28)$, and induction of the cytokines IFN $\gamma$, TNF $\alpha$, and IL-1 $\beta(20,29$

1. Abbreviations used in this paper: $\mathrm{AC}$, articular cartilage; CII, type II collagen; CIA, type II collagen-induced arthritis; CLN, collagenase; ECM, extracellular matrix; GLN-A, gelatinase A; GLN-B, gelatinase B; GAG, glycosaminoglycan; HC, hyaline cartilage; MMPs, matrix metalloproteinases; OA, osteoarthritic; PC, pericellular; PG, proteoglycan; PGIA, proteoglycan-induced arthritis; SLN, stromelysin. 
32). Of these, IL-1 $\beta$ appears to be very important because neutralizing antibodies ameliorate the histopathology and clinical symptoms in CIA (33-35) and because IL-1 $\beta$ induces SLN and CLN in putative target cells (36). SLN has been detected in CIA and in experimental arthritis induced by the intraarticular injection of IL-1 $\beta(22,37,38)$.

Because all MMPs are secreted as latent zymogens $(10,11$, 39,40 ) and the activities of mature MMPs are regulated by tissue inhibitors of MMPs (41-43), it is difficult to determine which of the expressed MMPs are active in RA. SLN and GLNA cleave aggrecan in the interglobular domain between amino acid residues $\mathrm{Asn}^{341}$ and $\mathrm{Phe}^{342}$, generating a hyaluronan-binding G1 fragment with the COOH-terminal sequence VDIPEN, which remains bound to hyaluronan in cartilage (7). Generation and accumulation of this VDIPEN neoepitope, which is common to both human and murine aggrecans $(44,45)$, can thus provide a convenient marker of MMP activity in cartilage. An antibody recognizing this $\mathrm{COOH}$-terminal aggrecan neoepitope has been developed; it does not recognize the VDIPEN sequence when it is an integral part of the peptide VDIPENFFGVG (46, 47). Because of this specificity, anti-VDIPEN IgG does not recognize intact aggrecan but does detect the G1 fragment generated by SLN or GLN-A. The purpose of our experiments was to determine whether the VDIPEN neoepitope is induced and localized in articular cartilage lesions as an early indicator of MMP activity in autoimmune murine models of inflammatory arthritis.

\section{Methods}

Animals. CIA was generated in B10.RIII mice (bred at Mayo Clinic) by injecting a single dose of highly purified porcine CII in CFA subcutaneously, as previously described (48). Control animals were injected with CFA emulsified with an equal volume of acid extraction buffer. PGIA was induced in female BALB/c mice (Charles River, Portage, MI) by intraperitoneal injection of chondroitinase ABC-digested canine aggrecan in CFA, followed by boosting with PG in incomplete Freund's adjuvant during weeks 1 and 4 . Controls were injected in the same manner but without antigen (18). CIA mice were killed $10 \mathrm{~d}$ after immunization and at day 20 when they showed stage 2 clinical signs ( $>3$ swollen toes per paw). PGIA animals were killed when the legs exhibited swollen digits for periods of 1-5 mo. Paws were removed above the ankle or wrist, quenched in $\mathrm{LN}_{2}$, and stored at $-70^{\circ} \mathrm{C}$. Animals were maintained in American Association of Laboratory Animal Care accredited facilities, and the experiments were approved by the respective institutional animal care and use committees.

Anti-VDIPEN antibody. An antibody was prepared against a peptide conjugate corresponding to the carboxy-terminal sequence of the MMPgenerated aggrecan G1 fragment (FVDIPEN ${ }^{341}$ ) and characterized (47). Peptide mapping studies using this antibody in an RIA indicate that it requires the free carboxyl group of the $\mathrm{COOH}$-terminal Asn for optimal recognition. If the $\mathrm{COOH}$-terminal Asn is either removed from the sequence, substituted with closely related amino acids, or extended across the MMP cleavage site, there is a $40-10,000$-fold loss in detection sensitivity. Further, this VDIPEN antibody detects an aggrecan G1 fragment with an $M_{\mathrm{r}}$ of 50,000 that is generated by the MMPs SLN and GLN-A using RIA or by Western blotting. In contrast, intact aggrecan is not recognized by this antibody. Under identical conditions, the closely related MMPs GLN-B and CLN, as well as cathepsin G, cathepsin B, and human leukocyte elastase, did not generate a G1 fragment recognized by this antibody.

Western blot analysis of aggrecan fragments. The skin and superficial muscle were dissected from mouse paws, and the remaining tissues were homogenized in buffer (see below) with or without $100 \mu \mathrm{g}$ of human recombinant $\mathrm{SLN}$, overnight at $37^{\circ} \mathrm{C}$. Active SLN was generated from recombinant human prostromelysin $(2 \mathrm{mM})$ in $25 \mathrm{mM}$ Tris- $\mathrm{HCl}$,

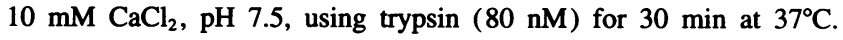
Trypsin was then inhibited with soybean trypsin inhibitor-agarose for $15 \mathrm{~min}$ at room temperature and the soybean trypsin inhibitor-agarose bound trypsin was removed from the sample by centrifugation. After digestion with SLN, aggrecan fragments were extracted from the homogenate using $4 \mathrm{M}$ guanidine hydrochloride in $10 \mathrm{mM}$ EDTA, $0.1 \mathrm{M}$ 6-aminohexanoic acid, $50 \mathrm{mM}$ benzamidine hydrochloride, $1 \mathrm{mM}$ phenylmethyl sulfonylfluoride, $50 \mathrm{mM} \mathrm{N}$-ethylmaleimide, and $1 \mathrm{mg} / \mathrm{ml}$ pepstatin. The extract was centrifuged at $3,000 \times g$ for $30 \mathrm{~min}$ at $4^{\circ} \mathrm{C}$. The supernatent containing the aggrecan was brought to $50 \mu \mathrm{g} / \mathrm{ml}$ with human umbilical cord hyaluronan (ICN Biologicals, Costa Mesa, CA) and dialyzed in a $3,000 \mathrm{~mol}$ wt cutoff membrane for $24 \mathrm{~h}$ at $4^{\circ} \mathrm{C}$ against $0.1 \mathrm{M}$ sodium acetate, $\mathrm{pH} 6.0$, containing the above proteinase inhibitors. After dialysis, the sample was centrifuged and the aggrecan/hyaluronan complex containing supernatent fractionated through an associative cesium chloride density gradient (starting density $1.5 \mathrm{gm} / \mathrm{ml}$ ) (49). The bottom fourth of the gradient (A1) was harvested and digested with protease-free chondroitinase $\mathrm{ABC}$ and keratanase II (Seigagaku America, Rockville, MD) as follows. Samples ( $100 \mu \mathrm{l})$ in $0.1 \mathrm{M}$ sodium acetate buffer, $\mathrm{pH} 8.0$, were brought to $10 \mathrm{mM}$ EDTA and treated with $0.02 \mathrm{U}$ protease-free chondroitinase $\mathrm{ABC}$ overnight at $37^{\circ} \mathrm{C}$. Keratanase II $(0.1 \mathrm{U})$ was then added to each sample and incubated at $37^{\circ} \mathrm{C}$ for 2 h. Samples ( 1 paw per lane) were then electrophoresed through 4-20\% SDS polyacrylamide Tris-glycine gels under reducing conditions and transferred to nitrocellulose. To eliminate nonspecific binding of antibodies, the nitrocellulose was incubated in 5\% nonfat dry milk for $1 \mathrm{~h}$ at room temperature. The membranes were incubated in a 1:3,000 dilution of anti-VDIPEN antiserum for $1 \mathrm{~h}$ at room temperature. The membranes were then washed and incubated with a 1:1,000 dilution of biotinylated-goat anti-rabbit IgG for $1 \mathrm{~h}$ at room temperature. Blots were washed and incubated with 1:1,000 dilution of alkaline phosphatasestreptavidin followed by 5-bromo-4-chloro-3-indolyl phosphate nitroblue tetrazolium (Kirkegaard and Perry, Inc., Gaithersburg, MD) to detect immunoreactive bands.

Immunoperoxidase microscopy. Cryofixation was selected as the primary fixation procedure to minimize epitope denaturation that usually accompanies chemical cross-linking and to limit PG extraction resulting from prolonged exposure of cartilage to aldehyde fixatives (50). Midsagittal 5- $\mu \mathrm{m}$ cryostat sections were cut through the fully calcified unfixed hind paws with a carbide knife. Cryosections were then mildly fixed for $\mathbf{2 0}$ min with Nakane solution (periodate/lysine/paraformaldehyde) (51) and treated with $3 \% \mathrm{H}_{2} \mathrm{O}_{2}$ in methanol to inactivate endogenous peroxidases, followed by $0.1 \%$ Triton-X 100 in PBS to increase permeability. To facilitate antibody penetration into cartilage (52), some sections were also digested with protease-free chondroitinase $\mathrm{ABC}(0.02$ $\mathrm{U}, 30 \mathrm{mM} \mathrm{Na}$ acetate, $0.1 \mathrm{M}$ Tris- $\mathrm{HCl}, \mathrm{pH} \mathrm{8.0;} \mathrm{Seigagaku} \mathrm{America)}$ before fixation. Because chondroitinase $\mathrm{ABC}$ has a pH optimum of 8.0 for removal of chondroitin sulfate from proteoglycans, whereas that for hyaluranan digestion is 6.0 , use of an alkaline $\mathrm{pH}$ greatly reduces hyaluronan cleavage and consequently circumvents possible release of aggrecan or aggrecan fragments from the sections (see Results). The VDIPEN neoepitope was labeled with rabbit anti-VDIPEN IgG (46) that was affinity-purified on VDIPEN-conjugated Reactigel (Pierce, Rockford, IL). An optimal staining concentration of $10 \mu \mathrm{g} / \mathrm{ml} \mathrm{IgG}$ was determined in titration experiments conducted on SLN-treated cryosections of normal murine paws. Bound antibodies were detected via immunoperoxidase microscopy using the $\mathrm{ABC}$ technique (Elite kit, Vector Labs., Inc., Burlingame, CA). Peroxidase reaction product was developed with a glucose oxidase/diaminobenzidine/nickel method to provide maximum sensitivity (53); $1 \%$ Orange $\mathrm{G}$ was used as a counterstain. For specificity controls, the primary antibody was incubated with either the neoepitope peptide $\left(\mathrm{H}_{2} \mathrm{~N}\right.$-YTGEDFVDIPEN-COOH) or a peptide that spans the neopitope cleavage site $\left(\mathrm{H}_{2} \mathrm{~N}-\mathrm{YTGEDFVDI}-\right.$ PENFFGV-COOH) and clarified before staining. Peptides were synthesized on an Applied Biosystems 430A peptide synthesizer and purified 
by reversed-phase HPLC on a Waters C18 Deltapak column. All peptides were $>95 \%$ pure by reversed-phase HPLC, and the structure of each was confirmed by electrospray ionization mass spectrometry. Preimmune rabbit IgG served as an additional negative control. For positive controls, unfixed paw cryosections were digested with activated recombinant human SLN $(100 \mu \mathrm{g} / \mathrm{ml}$ in $150 \mathrm{mM} \mathrm{NaCl}, 25 \mathrm{mM}$ Tris$\mathrm{HCl}, 10 \mathrm{mM} \mathrm{CaCl}, 0.05 \%$ Brij-35, $\mathrm{pH} \mathrm{7.4)} \mathrm{for} 30 \mathrm{~min}$ at $37^{\circ} \mathrm{C}$ before staining with anti-VDIPEN IgG. The cartilage GAG content of adjacent sections was also determined by toluidine blue staining as previously described (54). Photomicrographs were made at $10-25 \times$ with a Leitz Vario Orthomat microscope.

Image analysis. Induction of VDIPEN epitope and depletion of GAGs in CIA and PGIA mouse hind paw AC were measured semiquantitatively at $10-20 \times$ on a Zeiss Axioscope fitted with a Kodak Megaplus camera $(1,024 \times 1,024$ pixels $)$ using the Presage CV-6 digital image analysis system (Advanced Imaging, Princeton, $\mathrm{NJ}$ ). Areas exhibiting endogenous VDIPEN epitope were manually traced, and the mean pixel gray level (range of 0-255) of these regions was determined. Background densities, measured on adjacent sections stained using normal rabbit IgG as the primary antibody, were subtracted from each determination. Endogenous VDIPEN densities were expressed as a percent of the maximum VDIPEN staining obtained for uninvolved hyaline cartilage (HC) measured after exhaustive SLN digestion of adjacent sections.
Similarly, GAG depletion was expressed as the mean pixel density of toluidine blue-labeled HC divided by the pixel density of normal toluidine blue-stained HC observed in nearby sections.

\section{Results}

Anti-VDIPEN IgG specifically labels a neoepitope in SLNtreated normal mouse cartilage. Normal murine articular cartilage contains a very thin hyaline zone that is rich in GAGs but lacks detectable staining with anti-VDIPEN IgG, even after digestion of the cryosection with chondroitinase-ABC (Fig. 1, $A-C$ ). However, SLN digestion of unfixed mouse paw cryosections induces intense anti-VDIPEN staining within the GAGrich pericellular (PC) matrix of chondrocytes and at the articular cartilage surface (Fig. 1, $B$ and $D$ ); moderate VDIPEN labeling is also induced in the interterritorial zones of the AC (Fig. 1 D). Preincubation of anti-VDIPEN IgG with $250 \mathrm{ng} / \mathrm{ml}$ YTGEDFVDIPEN peptide completely blocked this labeling, whereas similar quantities of the spanning peptide (YTGEDFVDIPENFFGV) failed to inhibit staining (Fig. 1, $E$ and $F$ ). Sections of normal paws digested with chondroitinase-ABC followed by
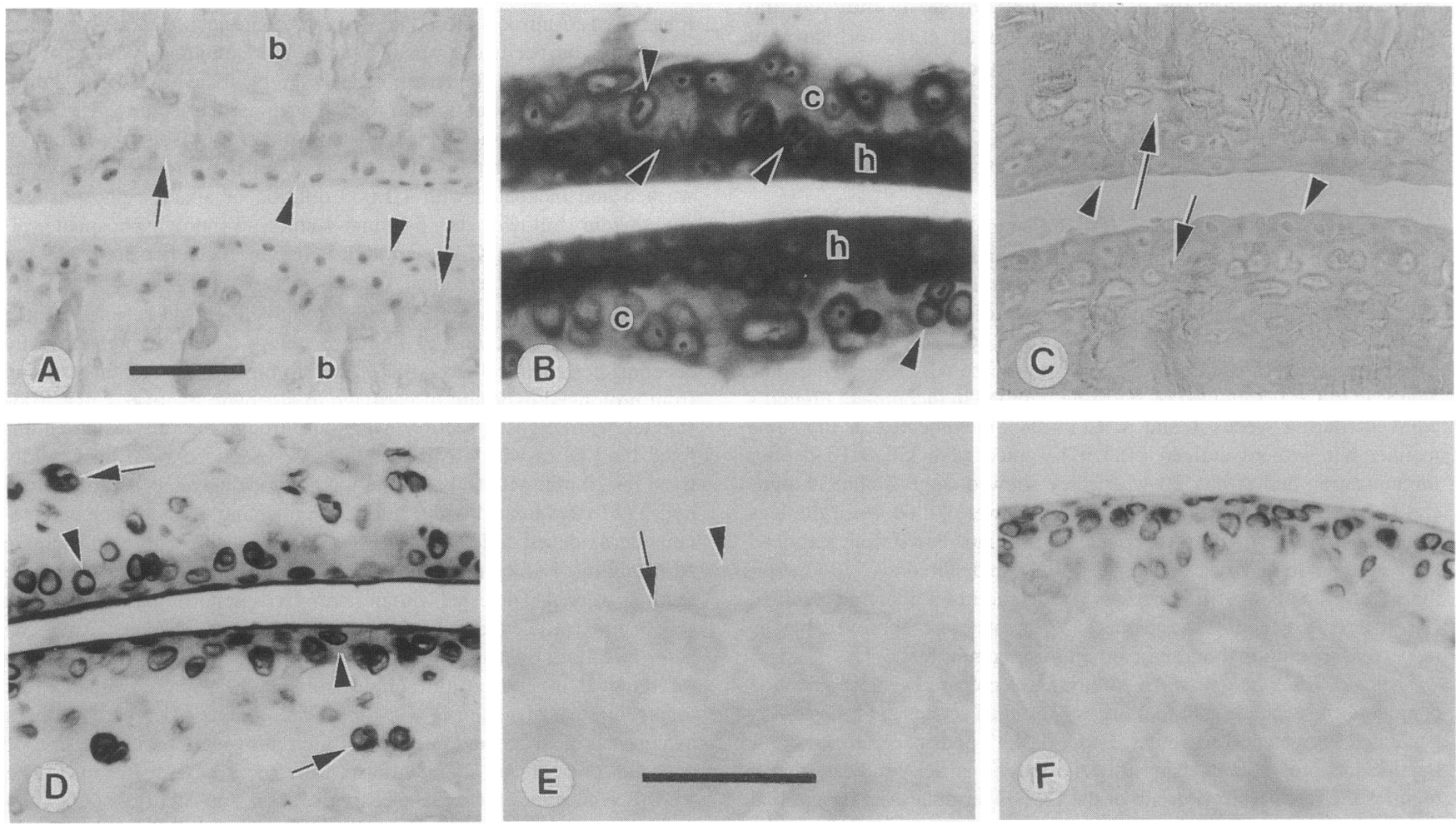

Figure 1. Anti-VDIPEN IgG specifically labels a SLN-induced neoepitope in cryosections of metatarsal articular cartilage from nonimmunized B10.R111 mice. ( $A$ ) Joints exhibit a thin hyaline layer (arrowheads) distal to a calcified zone (arrows) of articular cartilage and bone (b); $\mathrm{H} \&$ E staining. $(B)$ GAGs in the hyaline cartilage $(h)$ are intensely stained by toluidine blue, whereas the calcified cartilage $(c)$ shows moderate GAG staining. Chondrocytes in both AC layers also exhibit dense GAG labeling in their pericellular matrix (arrowheads). (C) No VDIPEN epitope is detected in the hyaline layer (arrowheads) or in the calcified zone (arrows) of normal AC stained with anti-VDIPEN IgG after chondroitinase$\mathrm{ABC}$ treatment; Orange-G counterstaining. $(D-F)$ Sections of normal paws were digested with SLN before immunostaining and counterstaining with Orange-G. $(D)$ Anti-VDIPEN IgG intensely stains the pericellular matrix of chondrocytes in the hyaline zone (arrowheads) and calcified zone (arrows) of the articular cartilage. The superficial region of the hyaline layer is also VDIPEN positive, and the territorial and interterritorial areas of the hyaline cartilage are moderately stained. $(E)$ No immunostaining is observed in the hyaline (arrowhead) and calcified (arrow) layers of AC if anti-VDIPEN IgG is preabsorbed with $250 \mathrm{ng} / \mathrm{ml}$ YTGEDFVDIPEN immunogen. $(F)$ Pretreatment of anti-VDIPEN IgG with $250 \mathrm{ng} / \mathrm{ml}$ of a peptide spanning the VDIPEN cleavage site (YTGEDFVDIPENFFGFG) does not prevent neoepitope labeling after SLN digestion of the cartilage. Bar for $A-D$ and $F, 50 \mu \mathrm{m}$; bar for $E, 50 \mu \mathrm{m}$. 


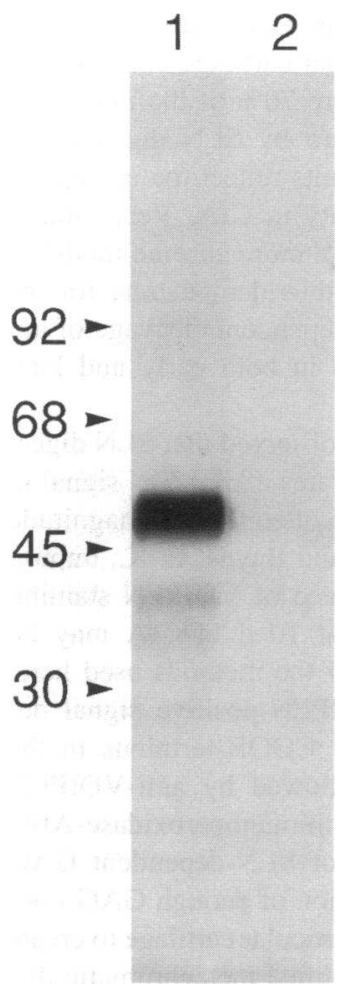

Figure 2. Anti-VDIPEN antibody detects a 50-kD aggrecan fragment in SLN-digested mouse paws. Extracts of paws from B10R.III mice were incubated with (lane 1) or without (lane 2) recombinant human SLN. The aggrecan fragments were extracted from the paw homoginate, fractionated through an associative cesium chloride density gradient, and evaluated by Western blotting using the anti-VDIPEN antibody. A single VDIPEN-positive band with a molecular mass of $50 \mathrm{kD}$ is observed only after SLN treatment of the paw extract.

SLN produced a similar pattern and density of VDIPEN labeling (not shown), indicating that chondroitinase-ABC does not cause a loss of putative MMP-cleavage sites (VDIPENFFGVG) within aggrecan. Also, a single 50-kD aggrecan G1 fragment was detected with this anti-VDIPEN antibody in Western blots of SLN-digested mouse paw extracts (Fig. 2). This size indicates that the entire sequence from the $\mathrm{NH}_{2}$-terminal Val through the $\mathrm{COOH}$-terminal $\mathrm{Asn}^{341}$ of the neoepitope has remained intact (7). The 50-kD aggrecan fragment maintained its ability to bind to hyaluronan as indicated by its sedimentation at the bottom of an associative cesium chloride density gradient when incubated with hyaluronan. The anti-VDIPEN antibody did not detect any other fragments in this digested sample, or in extracts of untreated control paws (Fig. 2), indicating that its ability to detect the $50-\mathrm{kD}$ aggrecan segment generated by SLN is specific. Together, these immunohistochemical and Western blotting results indicate that SLN generates the VDIPEN neoepitope on a single functional aggrecan G1 fragment in murine articular cartilage and that our antibody specifically detects this site only when its $\mathrm{COOH}$-terminus is exposed.

VDIPEN is induced and GAGs are depleted during CIA. CIA mouse paws exhibit a well-defined chronological series of gross clinical changes after immunization (48). Although no outward symptoms were visible at day 10 , many CIA mice showed redness and swelling in three or more digits of a given paw (clinical stage 2) by 15-20 d and ankylosis by day 28 . Most 10-, 20-, and 28-d CIA mice exhibited VDIPEN staining in their hind paw AC, whereas vehicle-injected controls were negative (Table I). However, the patterns of VDIPEN induction and GAG depletion differed markedly between 10 and $20 \mathrm{~d}$. Although limited foci of chondrocytes with pericellular VDIPEN labeling were observed in 10-d CIA mice (Fig. 3, $A$ -
Table I. Detection of the VDIPEN Neoepitope in Hind Paw Articular Cartilage of CIA Mice via Immunoperoxidase Microscopy

\begin{tabular}{lcr}
\hline \multicolumn{1}{c}{ Stage } & No. positive mice & Total \\
\hline 10 d postimmunization & 4 & 6 \\
$>$ 3 swollen digits per paw $(15-20 \mathrm{~d})$ & 13 & 14 \\
Ankylosis $(>28 \mathrm{~d})$ & 4 & 4 \\
Controls & 0 & 4
\end{tabular}

8-wk-old B10.RIII mice immunized intradermally with porcine CII in CFA. Midsagittal cryosections were cut through both hind paws of each animal.

C), GAG depletion of AC was not evident at this time. Levels of toluidine blue staining in AC were comparable with those of normal mice (data not shown, see Fig. $1 B$ ), and induced pericellular VDIPEN labeling was strikingly codistributed with high concentrations of PC GAGs (compare Fig. $3 B$ with Fig. $1 B$ ). Infiltrating inflammatory cells and cartilage erosion were also not detected at $10 \mathrm{~d}$.

In contrast, intense VDIPEN immunostaining was widespread at the articular surfaces of various tarsal and metatarsal joints of stage 2 paws at $20 \mathrm{~d}$ (Fig. 3, $D-K$ ). VDIPEN epitope appeared to be induced and heavily concentrated in the pericellular matrices of AC chondrocytes and at the eroding outer surface of the articular cartilage (Fig. 3, D, $I$, and $K$ ). Moderate VDIPEN labeling was also present in the interterritorial zones of AC (Fig. 3, $D$ and $I$ ). Induction of intense VDIPEN staining correlated with marked depletion of GAGs throughout the AC of adjacent cryosections at this stage of CIA (Fig. 3, $D$ and $E$ ). Erosion of the AC and infiltration with inflammatory cells and pannus were now very evident (Fig. $3, D-G$ ). The invading pannus sometimes showed strong VDIPEN staining (Fig. $3 G$ ), but it was often poorly labeled. Because intraarticular injection of SLN generates VDIPEN-bearing G1 fragments that are released into the synovial fluid (55), we believe that this synovial staining results from the differential uptake of VDIPEN fragments by macrophage-like cells of the pannus. Day 28 CIA specimens exhibited extensive cartilage and bone erosion, with small fragments of VDIPEN-positive AC remaining (not shown). These patterns of endogenous VDIPEN labeling were very similar to those observed after treatment of normal cartilage with SLN, except that endogenous VDIPEN immunostaining was not detected in the subadjacent calcified cartilage and bone (Figs. $1 D$, and 3, $D$ and $I$ ). SLN digestion of stage 2 paw cryosections did not diminish the VDIPEN labeling observed in damaged AC but induced VDIPEN neoepitope in the AC of other apparently normal joints present in the same section (as in Fig. $1 \mathrm{D}$ ). Predigestion of the sections with chondroitinase$A B C$ increased the staining intensity but did not alter the VDIPEN-staining pattern (not shown), indicating that this enzyme enhances accessibility of epitope to antibody and that it does not induce a loss of epitope-bearing aggrecan fragments. Endogenous VDIPEN staining was completely blocked by preincubating anti-VDIPEN IgG with YTGEDFVDIPEN (Fig. 3 $H$ ) but not by pretreatment with YTGEDFVDIPENFFGV (not shown); nonimmune rabbit IgG did not generate any immunoperoxidase labeling (Fig. $3 J$ ). This pattern of VDIPEN staining 
in CIA cartilage is highly specific, thus indicating that crossreactive epitopes are probably not being generated by other proteases during advanced disease.

Comparison with PGIA mice. Because PGIA appears to be a more chronic model than CIA, with waxing and waning clinical symptoms that progressively worsen 1-5 mo after immunization (18), experiments were also conducted on paws of PGIA mice. One month after disease onset, intense VDIPEN staining was present in AC (Fig. $4 \mathrm{~A}$ ) and was most concentrated in its pericellular matrix (Fig. $4 \mathrm{~B}$ ). Marked GAG depletion was present in corresponding $\mathrm{AC}$ regions observed in adjacent sections (Fig. $4 \mathrm{C}$ ). Similar patterns were observed after 5 mo of disease, whereas normal BALB/c mouse AC lacked significant endogenous VDIPEN epitope (not shown).

Morphometry of VDIPEN induction and GAG depletion. Semiquantitative histomorphometric measurements were performed using digital image analysis to compare the degrees of articular cartilage VDIPEN induction and GAG depletion observed between days 10 and 20 in CIA with corresponding levels found in PGIA (Fig. 5). Quantities of endogenous VDIPEN labeling were expressed as a percent of the maximum anti-VDIPEN signal generated by exhaustive SLN digestion of normal AC, and GAG depletion was normalized to the GAG content of untreated AC. Although the mean staining density observed in VDIPEN-positive foci at $10 \mathrm{~d}$ in CIA was $21 \%$ of the SLN-treated maximum, it increased to $70 \%$ of the maximum at $20 \mathrm{~d}$. GAG levels within corresponding AC regions were $95 \%$ of control values at day 10 but decreased to $38 \%$ of normal at $20 \mathrm{~d}$. Similar levels of VDIPEN induction and GAG depletion were observed in paw AC of mice with PGIA for 1-5 mo.

\section{Discussion}

We have shown that the VDIPEN ${ }^{341}$ aggrecan neoepitope is undetectable in normal mouse cartilage but is induced within $\mathrm{AC}$ of CIA mice as early as $10 \mathrm{~d}$ after immunization, in the complete absence of measurable GAG depletion, AC erosion, inflammatory infiltrate, and clinical symptoms. The VDIPEN neoepitope was concentrated in and colocalized with high concentrations of PGs in pericellular matrices of AC chondrocytes but was not detectable at the articular surface, in the interterritorial matrix, or in the calcified layer of cartilage at this time. SLN digestion of normal mouse paw cryosections also produced heavy PC VDIPEN labeling in both articular and calcified cartilage and moderate staining in interterritorial zones of the AC. This anti-VDIPEN staining is very specific because: $(a)$ it was inhibited by peptides with a VDIPEN ${ }^{341}$ COOH-terminus but not with peptides spanning the VDIPENFFGVG cleavage site, in both SLN-treated normal and arthritic AC; $(b)$ it was not induced when sections of normal paws were untreated or predigested with highly purified chondroitinase-ABC, a glycanase that removes chondroitin sulfate from aggrecan but does not

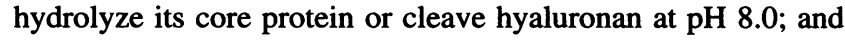
(c) a single 50-kD VDIPEN-positive fragment was detected upon digestion of paw extracts with SLN.

The levels of VDIPEN expression increased dramatically in stage 2 CIA paws by $20 \mathrm{~d}$ postimmunization; they were correlated with GAG depletion at this time, as shown by image analysis. Infiltration of inflammatory cells into the synovial cavity and the beginning of $\mathrm{AC}$ erosion were also very evident at this stage. Analysis of adjacent sections also showed that both induction of VDIPEN neoepitope and GAG depletion were strikingly colocalized at sites of articular cartilage damage. Because the induced VDIPEN levels were $70 \%$ of the maximum quantity of neoepitope created in vitro by SLN digestion of normal AC, we surmise that these results reflect the up-regulation of intense local enzymatic activity in CIA. Very similar observations were obtained for PGIA, a more chronic model of inflammatory arthritis. These studies thus demonstrate for the first time the semiquantitative MMP-dependent cleavage of aggrecan at sites of chondrodestruction in both early and later stages of arthritis.

Using image analysis, we recently observed that SLN digestion of normal paw cryosections generates a VDIPEN signal in AC more readily than a GAG depletion of equivalent magnitude could be produced (Donatelli, S. A., and Bayne, E. K., unpublished data). Thus, the early appearance of VDIPEN staining before overt GAG depletion found at $10 \mathrm{~d}$ in CIA may be due to differences in the sensitivity of the methods used here. Appearance of a SLN-mediated VDIPEN-positive signal depends on generation of the VDIPEN COOH-terminus in the interglobular region of aggrecan, followed by anti-VDIPEN staining and amplification via the immunoperoxidase-ABC technique. In comparison, detection of SLN-dependent GAG depletion requires cleavage and diffusion of enough GAG-containing aggrecan fragments out of the articular cartilage to create a reduction in staining with an unamplified metachromatic dye (toluidine blue). It is therefore possible that some GAG depletion also occurs in the early stages of AC damage in CIA but may not be as readily detectable as VDIPEN induction.

When the MMPs SLN, CLN, GLN-A, and GLN-B were evaluated for VDIPEN generation using intact aggrecan as a substrate, only SLN and GLN-A generated an epitope recognized by VDIPEN antibody; non-MMPs did not produce a VDIPEN signal $(46,47)$. However, other MMPs such as GLN$B$, interstitial or neutrophil CLN, and PUMP may also generate a VDIPEN COOH-terminus using a proteolyticially derived G1-G2 fragment of porcine aggrecan as a substrate (56-58). GLN-A is constituitively expressed in normal human synovia and is not up-regulated in disease, whereas SLN is dramatically induced in both RA and OA. These observations suggest that SLN may play a more dominant role than GLN-A in aggrecan catabolism in human arthritis (14). However, induction of VDIPEN staining in CIA and PGIA may reflect up-regulation of SLN and GLN-A activity during the early phases of matrix destruction. The question of whether one or both of these MMPs participate in VDIPEN induction may be answered when inhibitors specific for selected MMPs are developed and evaluated in these models of inflammatory arthritis.

These results do not rule out the possibility that additional enzymes could also be participating in aggrecan cleavage in CIA and PGIA. Recently, fragments consistent with another aggrecan cleavage site in the interglobular domain between $\mathrm{Glu}^{373}$ and $\mathrm{Ala}^{374}$ have been reported to accumulate in joint fluids of patients with inflammatory and noninflammatory joint diseases $(59,60)$. An enzyme, refered to as aggrecanase, has been proposed to generate this cleavage. We have prepared an antibody against the COOH terminus (NITEGE ${ }^{373}$ ) of this additional G1 fragment (61) that would result from "aggrecanase" cleavage and have detected it in articular cartilage of animals with CIA (62). When reagents become available to other specific aggrecan cleavage fragments, it will be interesting 

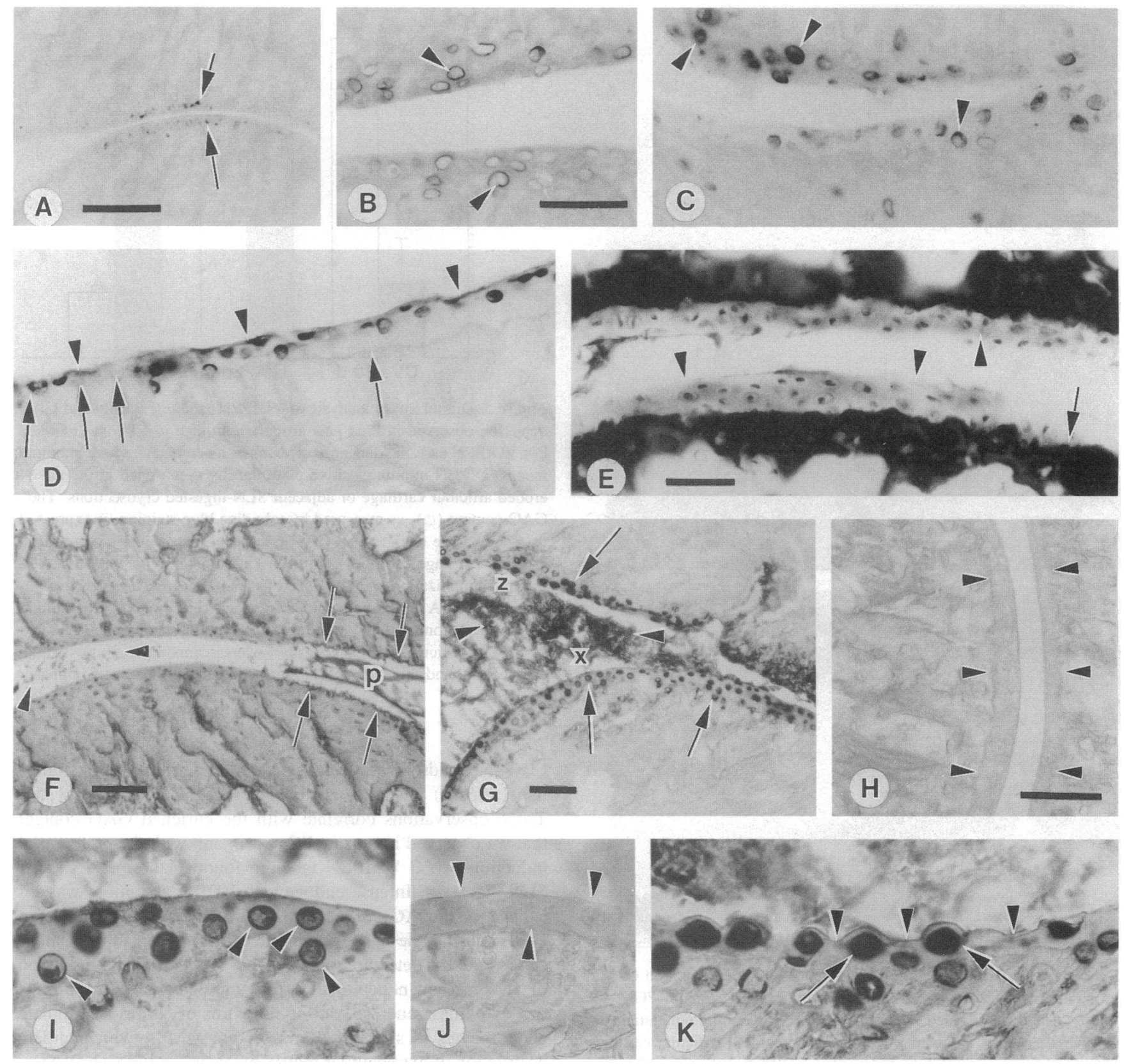

Figure 3. Distribution of endogenous VDIPEN neopitope and GAGs in hind paw articular cartilage from CIA mice. $(A-C)$ cryosections of tibiotarsal joints $10 \mathrm{~d}$ after immunization; $(D-K)$ sections of clinical stage 2 specimens. $(A-D, G, I$, and $K$ ) labeled with anti-VDIPEN IgG without chondroitinase-ABC pretreatment; $(H)$ stained with absorbed anti-VDIPEN IgG and $(I)$ with preimmune IgG; all were counterstained with Orange$\mathrm{G} ; E$ was stained with toluidine blue, and $F$ with $\mathrm{H} \& \mathrm{E}$. (A) Articular cartilage shows focal VDIPEN induction (arrows). (B) Delicate VDIPEN staining is limited to the pericellular matrix of AC chondrocytes (arrowheads). (C) Occasional foci show intense pericellular VDIPEN labeling (arrowheads) with apparent chondrocyte staining (middle arrowhead). (D) Stage 2 specimen exhibits erosion of the articular surface down to the calcified layer (arrows at left); the eroded surface and superficial pericellular matrix show intense VDIPEN staining (arrowheads). (E) Adjacent section depicts GAG depletion from the hyaline layer (arrowheads) and erosion of the articular surface (arrow). $(F)$ Metatarsal joint of stage 2 sample with inflammatory cells (arrowheads) and pannus $(p)$ in the synovial space and extensive articular cartilage erosion (arrows). $(G)$ Adjacent section showing VDIPEN accumulation in the articular cartilage (arrows) and pannus (arrowheads). $(H)$ Metatarsal joint from a paw with stage 2 CIA stained with anti-VDIPEN IgG that was preabsorbed using $250 \mathrm{ng} / \mathrm{ml}$ of the peptide YTGEDFVDIPEN. Layers of articular cartilage (arrowheads) and the subchondral bone are completely unlabeled. (I) Higher magnification of area $(X)$ in $G$ shows intense VDIPEN labeling of the articular cartilage PC matrix (arrowheads), the articular surface, and moderate staining of AC interterritorial zones. (J) VDIPEN staining is absent from articular cartilage (arrowheads) of an adjacent section treated with preimmune $\operatorname{IgG}$. $(K)$ Higher magnification of region $(z)$ in $G$ exhibits erosion of the articular surface (arrowheads) and intense VDIPEN staining of chondrocytes and associated pericellular matrix (arrows). Bar for $A, 200 \mu \mathrm{m}$; bar for $B-D$ and $I-K, 50 \mu \mathrm{m}$; bar for $E, 25 \mu \mathrm{m}$; bars for $F$ and $H, 100 \mu \mathrm{m}$; bar for $G, 50 \mu \mathrm{m}$. 

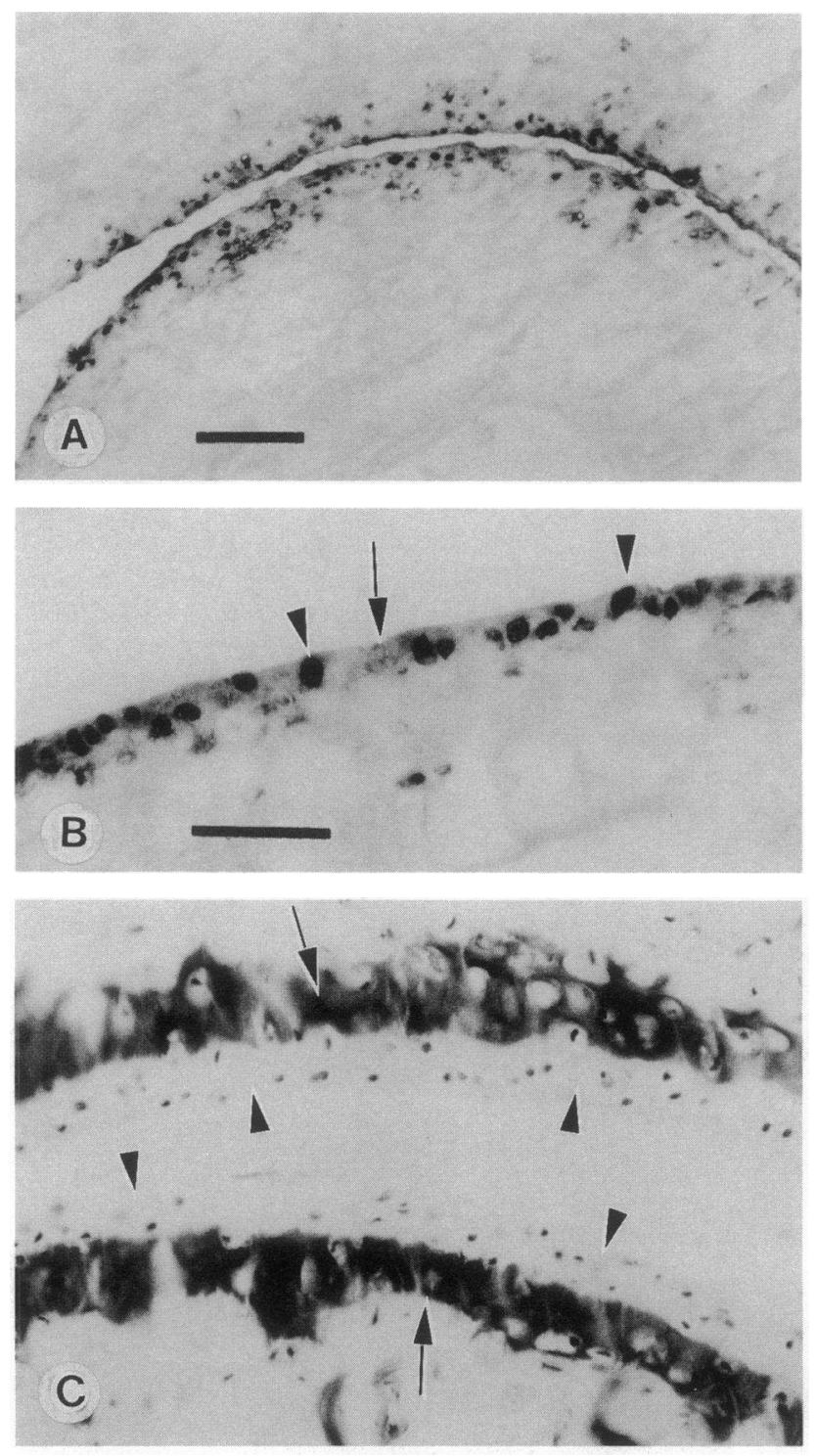

Figure 4. Distribution of endogenous VDIPEN neoepitope and GAGs in paw articular cartilage of mice exhibiting clinical signs of PGIA for 1 mo. ( $A$ and $B$ ) Labeled with anti-VDIPEN IgG and counterstained with Orange-G; $(C)$ stained with toluidine blue. $(A)$ Articular cartilage of a tibiotarsal joint exhibits widespread VDIPEN induction. $(B)$ VDIPEN staining is concentrated in the chrondrocytes and adjacent pericellular matrix of the articular cartilage (arrowheads), whereas the interterritorial matrix (arrow) is moderately labeled. (C) Articular cartilage ECM (arrowheads) shows marked depletion of GAGs, whereas the calcified layer (arrows) exhibits a normal level of GAG staining. Bar for $A, 100 \mu \mathrm{m}$; bar for $B$ and $C, 50 \mu \mathrm{m}$.

to localize them and to determine the time course during which the enzymes they represent may play a role in aggrecan cleavage. In any case, our observation of VDIPEN induction is the first to localize any specific aggrecan degradation product in an arthritis model and is the most direct evidence that MMP-mediated aggrecan degradation does take place in situ in CIA and PGIA. At minimum, our observation of VDIPEN induction is the most specific evidence for the involvement of MMP activity in animal models of arthritis available to date.

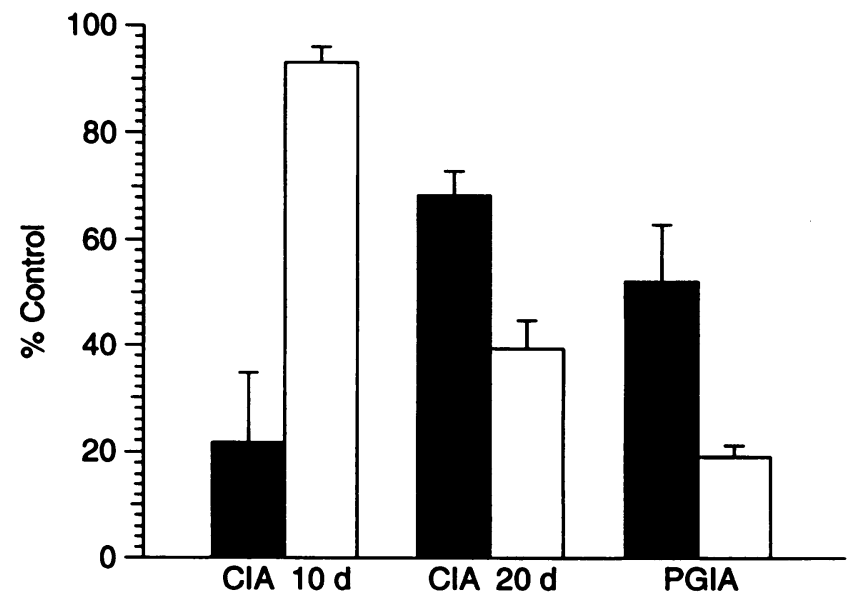

Figure 5. Digital image analysis of VDIPEN-labeling density and GAG depletion observed in hind paw articular cartilage in CIA and PGIA. For VDIPEN $(\square)$, the data are expressed as a percentage of the maximal mean VDIPEN immunopositive pixel density determined in the uneroded articular cartilage of adjacent SLN-digested cryosections. The GAG content ( $\square$ ), as measured by toluidine blue staining, is expressed as a percentage of the mean GAG gray-level pixel density in normal articular cartilage. Both hind paws of CIA animals killed at 10 and 20 $\mathrm{d}$ after immunization were analyzed ( $n=4$ and 7 mice, respectively). Similarly, PGIA data were obtained from a pool of three mice exhibiting clinical symptoms for $1 \mathrm{mo}$ and from one animal symptomatic for 5 mo. 10-12 articular cartilage surfaces were scanned per $5 \mu \mathrm{m}$ sagittal cryosection, and three sections were made per paw; brackets indicate the SEM.

The chondrocyte PC matrix is rich in aggrecan and hyaluronan $(50,63)$ and also exhibits elevated PG synthesis (35). These observations correlate with the enriched GAG content that we observed in murine PC matrix and with the preferential induction of PC VDIPEN labeling found in normal AC after SLN digestion. Intense endogenous VDIPEN staining was localized in the AC chrondrocyte PC matrix in both CIA and PGIA, during the early phases of disease when inflammatory cells were not detectable in the synovial cavity. The induction of an aggrecan neoepitope at this strategic location suggests that cytokines generated during inflammatory arthritis stimulate chondrocytes to synthesize and secrete MMPs, which initiate destruction of extracellular matrix by cleaving aggrecan. Therefore, the early appearance of VDIPEN labeling in PC matrix is a highly sensitive indicator of the commencement of joint pathology initiated by chondrocytes. Additional chondrocytegenerated proteases not detected by our VDIPEN antibody also participate in aggrecan and CII degradation within the pericellular matrix $(52,60,62)$. The later appearance of VDIPEN labeling at the articular surface further suggests that MMPs originating from the synovial cavity and pannus may also participate in destruction of articular cartilage. Other ECM-degrading proteases secreted by inflammatory cells could also increase accessibility of the superficial AC to MMPs, thus enhancing further VDIPEN labeling at the articular surface. The recent localization of VDIPEN staining in damaged regions of human RA and OA cartilage also indicates that the VDIPEN neopitope is a relevant marker of MMP-mediated aggrecan catabolism in human disease (64). Analogous observations have also been reported for degradation of $\mathrm{CII}$ in organ culture and arthritic 
human cartilage (52). Further studies of animal and arthritic patient cartilage using immunocytochemical methods to detect aggrecan neoepitopes generated by active MMPs and other proteases as described here should clarify further the role of these enzymes in the chondrodestructive process.

\section{Acknowledgments}

We are obliged to Dr. V. L. Moore for helpful suggestions and to Dr. J. A. Schmidt for constant critical and stimulating discussion.

This research was supported by Merck Research Laboratories. Dr. Nabozny is supported by a postdoctoral fellowship from the Arthritis Foundation.

\section{References}

1. Wight, T. N., D. K. Heinegård, and V. C. Hascal. 1991. Proteoglycans: structure and function. In Cell Biology of Extracellular Matrix. E. D. Hay, editor. Plenum Press, New York. 45-78.

2. Hardingham, T. E., and A. J. Fosang. 1992. Proteoglycans: many forms and many functions. FASEB (Fed. Am. Soc. Exp. Biol.) J. 6:861-870.

3. Birkedal-Hansen, H., W. G. I. Moore, M. K. Bodden, L. J. Windsor, H. B. Birkedal, A. Decarlo, and J. A. Engler. 1993. Matrix metalloproteinases: a review. Crit. Rev. Oral. Biol. Med. 4:197-250.

4. Matrisian, L. M. 1990. Metalloproteinases and their inhibitors in matrix remodeling. Trends Genet. 6:121-125.

5. Woessner, J. F., Jr. 1991. Matrix metalloproteinases and their inhibitors in connective tissue remodeling. FASEB (Fed. Am. Soc. Exp. Biol.) J. 5:2145-2154.

6. Murphy, G., and J. J. Reynolds. 1993. Extracellular matrix degradation. In Connective Tissue and Its Heritable Disorders. P. M. Royce and B. Steinmann, editors. Wiley-Liss, New York. 287-316.

7. Flannery, C. R., M. W. Lark, and J. D. Sandy. 1992. Identification of a stromelysin cleavage site within the interglobular domain of human aggrecan: evidence for proteolysis at this site in-vivo in human articular cartilage. J. Biol. Chem. 267:1008-1014.

8. Nguyen, Q., G. Murphy, P. J. Roughley, and J. S. Mort. 1989. Degradation of proteoglycan aggregate by a cartilage metalloproteinase: evidence for the involvement of stromelysin in the generation of link protein heterogeneity in situ. Biochem. J. 259:61-68.

9. Wu, J. J., M. W. Lark, L. E. Chun, and D. R. Eyre. 1991. Sites of stromelysin cleavage in collagen types ii, ix, x, and xi of cartilage. J. Biol. Chem. 266:56255628.

10. Walakovits, L. A., V. L. Moore, N. Bhardwaj, G. S. Gallick, and M. W. Lark. 1992. Detection of stromelysin and collagenase in synovial fluid from patients with rheumatoid arthritis and posttraumatic knee injury. Arthritis Rheum. 35:35-42.

11. Lohmander, L. S., L. A. Hoerrner, and M. W. Lark. 1993. Metalloproteinases, tissue inhibitor, and proteoglycan fragments in knee synovial fluid in human osteoarthritis. Arthritis Rheum. 36:181-189.

12. Okada, Y., N. Takeuchi, K. Tomita, I. Nakanishi, and H. Nagase. 1989. Immunolocalization of matrix metalloproteinase 3 (stromelysin) in rheumatoid synovioblasts (B cells): correlation with rheumatoid arthritis. Ann. Rheum. Dis. 48:645-653.

13. Okada, Y., M. Shinmei, O. Tanaka, K. Naka, A. Kimura, I. Nakanishi, M. T. Bayliss, K. Iwata, and H. Nagase. 1992. Localization of matrix metalloproteinase 3 (stromelysin) in osteoarthritic cartilage and synovium. Lab. Invest. 66:680-690.

14. Hutchinson, N. I., G. C. Wolfe, K. L. Macnaul, J. Martel-Pelletier, J. P. Pelletier, and F. F. Beuchel. 1994. Gelatinase-A mRNA is constitutively expressed in normal, OA, and RA synovial membranes. 40th Annual Meeting. Orthopedic Research Society, New Orleans, LA. 141. (Abstr.)

15. Wooley, P. H., H. S. Luthra, J. M. Stuart, and C. S. David. 1981. Type II collagen induced arthritis in mice. 1. Major histo compatibility complex I region linkage and antibody correlates. J. Exp. Med. 154:688-700.

16. Wooley, P. H., A. M. Dillon, H. S. Luthra, J. M. Stuart, and C. S. David. 1983. Genetic control of type-II collagen-induced arthritis in mice: factors influencing disease susceptibility and evidence for multiple mhc-associated gene control. Transplant. Proc. 15:180-185.

17. Griffiths, M. M., E. J. Eichwald, J. H. Martin, C. B. Smith, and C. W. DeWitt. 1981. Immunogenetic control of experimental type II collagen induced arthritis. 1. Susceptibility and resistance among inbred strains of rats. Arthritis Rheum. 24:781-789.

18. Glant, T. T., K. Mikecz, A. Arzoumanian, and A. R. Poole. 1987. Proteo- glycan-induced arthritis in BALB/c mice. Clinical features and histopathology. Arthritis Rheum. 30:201-212.

19. Glant, T. T., K. Mikecz, E. Buzas, E. Dayer, and A. R. Poole. 1991 Antiproteoglycan antibodies in experimental spondylarthritis. In Monoclonal Antibodies, Cytokines, and Arthritis. Mediators of Inflammation and Therapy. T. Kresina, editor. Marcel Dekker, Inc., New York. 341-356.

20. Glant, T. T., K. Mikecz, E. J. M. A. Thonar, and K. E. Kuettner. 1993. Immune responses to cartilage proteoglycans in inflammatory animal models and human diseases. In Cartilage Degradation: Basic and Clinical Aspects. J. F. Woessner and D. S. Howell, editors. Marcel Dekker, Inc., New York. 435-473.

21. Mikecz, K., T. T. Glant, and A. R. Poole. 1987. Immunity to cartilage proteoglycans in BALB/c mice with progressive polyarthritis and ankylosing spondylitis induced by injection of human cartilage proteoglycan. Arthritis Rheum. 30:306-318.

22. Hasty, K. A., R. A. Reife, A. H. Kang, and J. M. Stuart. 1990. The role of stromelysin in the cartilage destruction that accompanies inflammatory arthritis. Arthritis Rheum. 33:388-397.

23. Watson, W. C., and A. S. Townes. 1985. Genetic susceptibility to murine collagen ii autoimmune arthritis: proposed relationship to the immunoglobulin g-2 autoantibody subclass response, complement c-5, major histocompatibility complex, and non-major histocompatibility complex. J. Exp. Med. 162:18781891.

24. Spinella, D. G., J. R. Jeffers, R. A. Reife, and J. M. Stuart. 1991. The role of $\mathrm{c} 5$ and $\mathrm{t}$-cell receptor $\mathrm{v} \beta$ genes in susceptibility to collagen-induced arthritis. Immunogenetics. 34:22-27.

25. Reife, R. A., N. Loutis, W. C. Watson, K. A. Hasty, and J. M. Stuart. 1991. Swr mice are resistant to collagen-induced arthritis but produce potentially arthritogenic antibodies. Arthritis Rheum. 34:776-781.

26. Banerjee, S., B. Y. Wei, K. Hillman, H. S. Luthra, and C. S. David. 1988. Immunosuppression of collagen-induced arthritis in mice with an anti-II-2 receptor antibody. J. Immunol. 141:1150-1154.

27. Hom, J. T., L. D. Butler, P. E. Riedl, and A. M. Bendele. 1988. The progression of the inflammation in established collagen-induced arthritis can be altered by treatments with immunological or pharmacological agents which inhibit T cell activities. Eur. J. Immunol. 18:881-888.

28. Moder, K. G., H. S. Luthra, R. Kubo, M. Griffiths, and C. S. David. 1992. Prevention of collagen induced arthritis in mice by treatment with an antibody directed against the T cell receptor alpha beta framework. Autoimmunity. 11:219_ 224.

29. Williams, R. O., M. Feldmann, and R. N. Maini. 1992. Anti-tumor necrosis factor ameliorates joint disease in murine collagen-induced arthritis. Proc. Natl. Acad. Sci. USA. 89:9784-9788.

30. Thorbecke, G. J., R. Shah, C. H. Leu, A. P. Kuruvilla, A. M. Hardison, and M. A. Palladino. 1992. Involvement of endogenous tumor necrosis factor alpha and transforming growth factor beta during induction of collagen type II arthritis in mice. Proc. Natl. Acad. Sci. USA. 89:7375-7379.

31. Mauritz, N. J., R. Holmdahl, R. Jonsson, D. M. P. H. Van, A. Scheynius, and L. Klareskog. 1988. Treatment with gamma interferon triggers the onset of collagen arthritis in mice. Arthritis Rheum. 31:1297-1304.

32. Hom, J. T., A. M. Bendele, and D. G. Carlson. 1988. In vivo administration with II-1 accelerates the development of collagen-induced arthritis in mice. $J$. Immunol. 141:834-841.

33. Geiger, T., H. Towbin, A. Cosenti-Vargas, O. Zingel, J. Arnold, C. Rordorf, M. Glatt, and K. Vosbeck. 1993. Neutralization of interleukin-1-beta activity in-vivo with a monoclonal antibody alleviates collagen-induced arthritis in DBA/ 1 mice and prevents the associated acute-phase response. Clin. Exp. Rheumatol. 11:515-522.

34. Van de Loo, F. A., O. J. Arntz, I. G. Otterness, and W. B. Van den Berg. 1992. Protection against cartilage proteoglycan synthesis inhibition by antiinterleukin 1 antibodies in experimental arthritis. J. Rheumatol. 19:348-356.

35. Van den Berg, W. B., L. A. Joosten, M. Helsen, and F. A. van de Loo. 1994. Amelioration of established murine collagen-induced arthritis with anti-IL1 treatment. Clin. Exp. Immunol. 95:237-243.

36. MacNaul, K. L., N. Chartrain, M. Lark, M. J. Tocci, and N. I. Hutchinson. 1990. Discoordinate expression of stromelysin, collagenase, and tissue inhibitor of metalloproteinases-1 in rheumatoid synovial fibroblasts. J. Biol. Chem. 265:17238-17245.

37. McDonnell, J., L. A. Hoerrner, M. W. Lark, C. Harper, T. Dey, J. Lobner, G. Eiermann, D. Kazazis, I. I. Singer, and V. L. Moore. 1992. Recombinan human interleukin-1-beta-induced increase in levels of proteoglycans, stromelysin. and leukocytes in rabbit synovial fluid. Arthritis Rheum. 35:799-805.

38. Hutchinson, N. I., M. W. Lark, K. L. Macnaul, C. Harper, L. A. Hoerrner, J. McDonnell, S. Donatelli, V. Moore, and E. K. Bayne. 1992. In-vivo expression of stromelysin in synovium and cartilage of rabbits injected intraarticularly with interleukin-1-beta. Arthritis Rheum. 35:1227-1233.

39. Van Wart, H. E., and H. H. Birkedal. 1990. The cysteine switch: a principle of regulation of metalloproteinase activity with potential applicability to the entire matrix metalloproteinase gene family. Proc. Natl. Acad. Sci. USA. 87:5578-5582. 
40. Wolfe, G. C., K. L. MacNaul, F. F. Buechel, J. McDonnell, L. A. Hoermer, M. W. Lark, V. L. Moore, and N. I. Hutchinson. 1993. Differential in vivo expression of collagenase messenger RNA in synovium and cartilage. Arthritis Rheum. 36:1540-1547.

41. Umenishi, F., M. Umeda, and K. Miyazaki. 1991. Efficient purification of TIMP-2 from culture medium conditioned by human hepatoma cell line and its inhibitory effects on metalloproteinases and in vitro tumor invasion. J. Biochem. 110:189-195.

42. Yasumitsu, H., K. Miyazaki, F. Umenishi, N. Koshikawa, and M. Umeda. 1992. Comparison of extracellular matrix-degrading activities between 64-kda and 90-kda gelatinases purified in inhibitor-free forms from human schwannoma cells. J. Biochem. 111:74-80.

43. Goldberg, G. I., B. L. Marmer, G. A. Grant, A. Z. Eisen, S. Wilhelm, and C. He. 1989. Human 72-kilodalton type IV collagenase forms a complex with a tissue inhibitor of metalloproteases designated TIMP-2. Proc. Natl. Acad. Sci. USA. 86:8207-8211.

44. Doege, K. J., M. Sasaki, T. Kimura, and Y. Yamada. 1991. Complete coding sequence and deduced primary structure of the human cartilage large aggregating proteoglycan, aggrecan. Human-specific repeats, and additional alternatively spliced forms. J. Biol. Chem. 266:894-902.

45. Walcz, E., F. Deak, P. Erhardt, S. N. Coulter, C. Fulop, P. Horvath K. J. Doege, and T. T. Glant. 1994. Complete coding sequence, deduced primary structure, chromosomal localization, and structural analysis of murine aggrecan Genomics. 22:364-371.

46. Lark, M., H. Williams, L. Hoerrner, J. Weidner, C. Harper, J. Ayala, A Christen, J. Olszewski, Z. Konteatis, and R. Mumford. 1994. Development of an antibody against a metalloproteinase generated neo-epitope in human aggrecan 40th Annual Meeting, Orthopedic Research Society, New Orleans, LA. 313. (Abstr.)

47. Lark, M. W., H. Williams, L. A. Hoerner, J. Weidner, J. Ayala, C. F. Harper, A. Christen, J. Olszewski, Z. Konteatis, and R. A. Mumford. 1995 Quantitation of a matrix metalloproteinase-generated aggrecan G1 fragment using a monospecific anti-peptide antiserum. Biochem. J. 307:245-252.

48. Wooley, P. H. 1988. Collagen-induced arthritis in the mouse. Methods Enzymol. 162:361-373.

49. Hascall, V. C., and J. H. Kimura. 1982. Proteoglycans, isolation and characterization. Methods Enzymol. 82:7692-7800.

50. Hunziker, E. B., and W. B. Hermann. 1987. In situ localization of cartilage extracellular matrix components by immunoelectron microscopy after cryotechnical tissue processing. J. Histochem. Cytochem. 35:647-655.

51. McLean, I. W., and P. K. Nakane. 1974. Periodate-lysine-paraformaldehyde fixative. A new fixation for immunoelectron microscopy. J. Histochem. Cytochem. 22:1077.

52. Dodge, G. R., and A. R. Poole. 1989. Immunohistochemical detection and immunochemical analysis of type II collagen degradation in human norma rheumatoid and osteoarthritic articular cartilages and in explants of bovine articular cartilage cultured with interleukin 1. J. Clin. Invest. 83:647-661.
53. Shu, S., G. Ju, and L. Fan. 1988. The glucose oxidase-DAB nickel method in peroxidase histochemistry of the nervous system. Neurosci. Lett. 85:169-171.

54. Getzy, L. L., C. J. Malemud, V. M. Goldberg, and R. W. Moskowitz. 1982. Factors influencing metachromatic staining in paraffin-embedded section of rabbit and human articular cartilage: a comparison of the Safranin $O$ and Toluidine blue $\mathrm{O}$ techniques. J. Histotechnol. 5:111-116.

55. Moore, V. L., J. Olszewski, J. McDonnell, C. Saphos, H. Williams, and R. Mumford. 1994. Elicitation of a stromelysin-specific neoepitope in the IGD region of aggrecan by the intraarticular injection of human stromelysin. 40th Annual Meeting, Orthopedic Research Society, New Orleans, LA. 312 (Abstr.)

56. Fosang, A. J., P. J. Neame, T. E. Hardingham, G. Murphy, and J. A. Hamilton. 1991. Cleavage of cartilage proteoglycan between $\mathrm{g} 1$ and $\mathrm{g} 2$ domains by stromelysins. J. Biol. Chem. 266:15579-15582.

57. Fosang, A. J., K. Last, V. Knauper, P. J. Neame, G. Murphy, T. E. Hardingham, H. Tschesche, and J. A. Hamilton. 1993. Fibroblast and neutrophi collagenases cleave at two sites in the cartilage aggrecan interglobular domain Biochem. J. 295:273-276.

58. Fosang, A. J., K. Last, P. J. Neame, C. E. Hughes, B. Caterson, T. E. Hardingham, V. Knauper, G. Murphy, and H. Tschesche. 1994. Neutrophil collagenase cleaves the aggrecanase site in the interglobular region of aggrecan. 40th Annual Meeting, Orthopedic Research Society, New Orleans, LA. 48. (Abstr.)

59. Sandy, J. D., C. R. Flannery, P. J. Neame, and L. S. Lohmander. 1992. The structure of aggrecan fragments in human synovial fluid. Evidence for the involvement in osteoarthritis of a novel proteinase which cleaves the $\mathrm{Glu}^{373}-\mathrm{Ala}^{374}$ bond in the interglobular domain. J. Clin. Invest. 89:1512-1516.

60. Lohmander, L. S., P. J. Neame, and J. D. Sandy. 1993. The structure of aggrecan fragments in human synovial fluid. Evidence that aggrecanase mediates cartilage degradation in inflammatory joint disease, joint injury, and osteoarthritis. Arthritis Rheum. 36:1214-1222.

61. Lark, M. W., J. T. Gordy, J. R. Weidner, J. Ayala, J. H. Kimura, H. R. Williams, R. A. Mumford, C. R. Flannery, S. S. Carlson, M. Iwata, et al. 1995 Cell mediated catabolism of aggrecan. Evidence that cleavage at the "aggrecanase" site $\left(\mathrm{Glu}^{373}-\mathrm{Ala}^{374}\right)$ is a primary event in proteolysis of the interglobular domain. J. Biol. Chem. 270:2550-2556.

62. Singer, I. I., S. Scott, D. W. Kawka, E. K. Bayne, S. A. Donatelli, J. R. Weidner, H. R. Williams, R. A. Mumford, M. W. Lark, J. McDonnell, et al. 1995 Aggrecanase and metalloproteinase-specific aggrecan neo-epitopes are induced in the articular cartilage of mice with collagen II arthritis. 41st Annual Meeting, Orthopedic Research Society, Orlando, FL. 330. (Abstr.)

63. Knudson, C. B. 1993. Hyaluronan receptor-directed assembly of chondrocyte pericellular matrix. J. Cell Biol. 120:825-834.

64. Bayne, E. K., S. A. Donatelli, I. I. Singer, J. R. Weidner, N. I. Hutchinson, L. A. Hoerner, H. R. Williams, R. A. Mumford, L. S. Lohmander, and M. W. Lark. 1994. Detection of a metalloproteinase-generated aggrecan HABR fragment within human OA and RA cartilage. 40th Annual Meeting, Orthopedic Research Society, New Orleans, LA. 308. (Abstr.) 